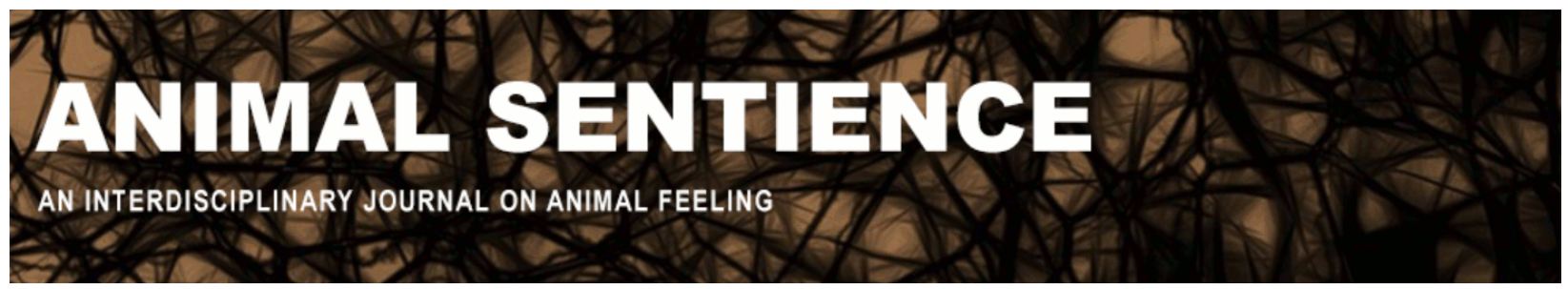

Johnsson, Martin (2017) Changes in behavior and emotion under chicken domestication. Animal Sentience 17(10)

DOI: $10.51291 / 2377-7478.1270$

Date of submission: 2017-12-04

Date of acceptance: 2017-12-05 (c) 


\title{
Changes in behavior and emotion under chicken domestication
}

\author{
Commentary on Marino on Thinking Chickens
}

\author{
Martin Johnsson \\ The Roslin Institute \\ Swedish University of Agricultural Sciences \& University of Edinburgh
}

\begin{abstract}
Marino's target article provides an overview of chickens' cognition, emotion, and personality, with the aim of changing how people view chickens. In this commentary, I will agree that chickens deserve better than their reputation, but contend with a statement about the lack of behavioral change under chicken domestication.
\end{abstract}

Martin Johnsson is a postdoctoral researcher who studies the genomics of chickens and pigs. www.alphagenes.roslin.ed.ac.uk /dr-martin-johnsson/

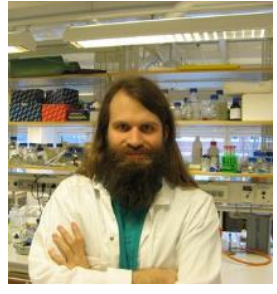

The target article by Marino $(2017 \mathrm{a}, \mathrm{b})$ provides an overview of chickens' cognition, emotion, and personality, with the aim of changing how people view chickens. When invited to comment, I asked myself what a geneticist could bring to this discussion. Behavior genetics deals with questions on a smaller scale. Not "what is chicken cognition, emotion, personality, and sociality like?" but rather "how do chickens vary, within and between populations, in those regards?" In this commentary, I will agree that chickens deserve better than their reputation, but I will challenge the statement about the lack of behavioral change under chicken domestication.

First, anecdotally, I agree that dismissive attitudes about chickens seem common in research too. "But chickens are not real birds, right?" said a serious evolutionary biologist, jokingly. It is a joke that betrays an attitude. If we look back at Price's (1984) review of behavior in domestication, he had to devote a whole section to refute the degeneration hypothesis.

It is sometimes claimed that domestic animals are not "real" animals. This belief is fostered by the notion that domestic animals are "degenerate," a concept that is largely derived from reports that many stocks of domestic animals are incapable of surviving the rigors of life in the wild and that in captivity many biological traits are lost through disuse. (Darwin, 1868)

Price rightly answers that domestication can be viewed as an adaptation to a particular environment, a life with humans, and that the ability to survive in the wild is irrelevant because "most domestic animals do not live in nature."

Domestication literature often claims that domestic animals have smaller brains than wild animals. This may be true (after all, post-mortem brain mass is not difficult to measure), but what does it mean? It matters whether we measure absolute or relative brain size. Broiler chickens have relatively smaller brains than Red Junglefowl (Jackson and Diamond 1996), a fact that the authors interpret as adaptive reduction of an energy-demanding but 
superfluous brain mass. White Leghorn layer chickens also have a relatively smaller, but absolutely larger, brain than Red Junglefowl (Henriksen et al. 2016). However, the increase in body mass is, as far as we can tell from genetic mapping, genetically independent of the loci that affect brain mass. So, it seems, domestic White Leghorn chickens have a smaller relative brain mass mostly because of their greater overall body mass, which is probably unrelated to their cognitive abilities.

It is probably correct that personality plays a part in human appreciation of animals. I think that is a regrettable bias. Animal personality, as understood by behavioral scientists, means little more than repeatable individual variation (Briffa and Weiss 2010). It would be unfortunate indeed if animals who have reduced inter-individual variation, for instance, as a consequence of breeding, would be seen as less worthy of our regard and protection for that reason.

I will disagree with one statement about chicken domestication, though. The review contrasts dogs and chickens, saying that the latter have not changed their behavior that much with domestication. This may be true for cognitive capacity and perception, but it is not the case for emotion and sociality.

First, although dogs are doubtlessly very skilled at interacting with humans, we do not really know how unique they are in their abilities. For example, horses (Malavasi and Huber 2016), goats (Nawroth et al. 2016), and experimentally domesticated foxes (Hare et al. 2005) are also able to perform some of the human-directed social tasks that dogs are good at. Are chickens? Maybe not, but the point is that domestication has wide-ranging effects on behavior, not just on companion animals. Both dogs and chickens are thought to have followed a similar course to becoming domesticated, namely, a period of coevolution with humans before becoming managed (Zeder 2012).

Second, domestic chickens differ from Red Junglefowl in several facets of their fearful, social, and foraging behaviors (Schütz et al. 2004; Schütz et al. 2001), as well as their stress responses (Ericsson et al. 2014; Ericsson and Jensen 2016; Fallahsharoudi et al. 2017). These studies survey a limited part of domestic and Junglefowl diversity, but they are in line with the hypothesis that fearful behavior is a central part of animal domestication. Selection for reduced fear of humans in Red Junglefowl in an artificial domestication study produces correlated changes in growth, and social dominance (Agnvall et al. 2014; Agnvall et al. 2012).

Third, unchanged behavior and emotionality would be anathema to the thinking in domestication genetics, where the hypothesis is that tameness is a driving force in domestication. Belyaev (1979) and coworkers performed the paradigmatic experiment, showing that selection for tameness rapidly produces domestic-like animals). This underpins the hypothesis that something about selection for tameness "destabilizes" other aspects of behavior, and causes correlated changes in domestication. Lately, this hypothesis has been embellished with speculations about epigenetics (Trut et al. 2009) and neural crest cells (Sánchez-Villagra et al. 2016; Wilkins et al. 2014), but the core idea that tameness is a driver of behavioral change in domestication persists. Domestic animals would not be domestic had their behavior not been changed.

Even today, chicken breeding is likely influencing social behavior and stress responses. (A minor quibble: Marino writes that domestic chickens are subject to "genetic manipulation" for production traits. This is, as far as I can tell, only true in the sense that artificial selection is genetic manipulation.) Chickens are selected to cope with large group sizes, and against feather pecking, a behavior problem connected with social interactions and stress (Brunberg et al. 2016). Regardless of whether these behaviors are part of the 
explicit breeding goal, natural selection will work on how well the chickens cope with their environment.

In summary, changes in emotionality and sociality are parts of domestication. It is true that chicken welfare is a more problematic than dog welfare, but not because chickens would be less adapted to life with humans.

\section{References}

Agnvall, B., A. Ali, S. Olby, and P. Jensen, 2014. Red Junglefowl (Gallus gallus) selected for low fear of humans are larger, more dominant and produce larger offspring. Animal 8 (09):1498-1505.

Agnvall, B., M. Jöngren, E. Strandberg, and P. Jensen, 2012. Heritability and genetic correlations of fear-related behaviour in Red Junglefowl-possible implications for early domestication. PLOS ONE 7 (4):e35162.

Belyaev, D.K., 1979. Destabilizing selection as a factor of domestication. Journal of Heredity 70:301-308.

Briffa, M., and A. Weiss, 2010. Animal personality. Current Biology 20 (21):R912-R914.

Brunberg, E.I., T.B. Rodenburg, L. Rydhmer, J.B. Kjaer, P. Jensen, and L. J. Keeling, 2016. Omnivores going astray: $A$ review and new synthesis of abnormal behavior in pigs and laying hens. Frontiers in Veterinary Science 3.

Ericsson, M., A. Fallahsharoudi, J. Bergquist, M.M. Kushnir, and P. Jensen, 2014. Domestication effects on behavioural and hormonal responses to acute stress in chickens. Physiology \& Behavior 133:161-169.

Ericsson, M., and P. Jensen, 2016. Domestication and ontogeny effects on the stress response in young chickens (Gallus gallus). Scientific Reports 6.

Fallahsharoudi, A., N. de Kock, M. Johnsson, L. Bektic, S.J.K.A. Ubhayasekera, J. Bergquist, D. Wright, and P. Jensen, 2017. Genetic and targeted eQTL mapping reveals strong candidate genes modulating the stress response during chicken domestication. G3: Genes, Genomes, Genetics 7 (2):497-504.

Hare, B., I. Plyusnina, N. Ignacio, O. Schepina, A. Stepika, R. Wrangham, and L. Trut, 2005. Social cognitive evolution in captive foxes is a correlated by-product of experimental domestication. Current Biology 15 (3):226-230.

Henriksen, R., M. Johnsson, L. Andersson, P. Jensen, and D. Wright, 2016. The domesticated brain: genetics of brain mass and brain structure in an avian species. Scientific Reports 6.

Jackson, S., and J. Diamond, 1996. Metabolic and digestive responses to artificial selection in chickens. Evolution 50 (4):1638-1650.

Malavasi, R., and L. Huber, 2016. Evidence of heterospecific referential communication from domestic horses (Equus caballus) to humans. Animal Cognition 19 (5):899-909.

Marino, L., 2017a. The inconvenient truth about thinking chickens. Animal Sentience 17(1).

Marino, L., 2017b. Thinking chickens: A literature review of cognition, emotion, and behavior in the domestic chicken. Animal Cognition 20 (2):127-141.

Nawroth, C., J.M. Brett, and A.G. McElligott, 2016. Goats display audience-dependent human-directed gazing behaviour in a problem-solving task. Biology Letters 12 (7):20160283.

Price, E.O., 1984. Behavioral aspects of animal domestication. Quarterly Review of Biology 59 (1):1-32. 
Sánchez-Villagra, M.R., M. Geiger, and R.A. Schneider, 2016. The taming of the neural crest: A developmental perspective on the origins of morphological covariation in domesticated mammals. Open Science 3 (6):160107.

Schütz, K., B. Forkman, and P. Jensen, 2001. Domestication effects on foraging strategy, social behaviour and different fear responses: a comparison between the red junglefowl (Gallus gallus) and a modern laying strain. Applied Animal Behaviour Science 74:1-14.

Schütz, K., S. Kerje, L. Jacobsson, B. Forkman, Ö. Carlborg, L. Andersson, and P. Jensen, 2004. Major growth QTLs in fowl are related to fearful behavior: Possible genetic links between fear responses and production traits in a red junglefowl $x$ White Leghorn intercross. Behavior Genetics 34:121-130.

Trut, L., I. Oskina, and A. Kharlamova, 2009. Animal evolution during domestication: The domesticated fox as a model. Bioessays 31 (3):349-360.

Wilkins, A.S., R.W. Wrangham, and W.T. Fitch, 2014. The "domestication syndrome" in mammals: A unified explanation based on neural crest cell behavior and genetics. Genetics 197 (3):795-808.

Zeder, M.A., 2012. The domestication of animals. Journal of Anthropological Research 68 (2):161-190. 